\title{
Trend analyses of hierarchical pin-point cover data
}

\author{
Christian DamgaARD ${ }^{1}$ \\ Bioscience, Aarhus University, Vejlsøvej 25, 8600 Silkeborg, Denmark
}

\begin{abstract}
The use of state-space models for analyzing longitudinal hierarchical pin-point plant cover data is demonstrated. The main advantages of using a state-space model are (1) that the observed variance is separated into sampling variance and the more interesting structural variance which are needed for quantifying prediction uncertainty, (2) that missing values or an unbalanced sampling design readily may be accounted for, and (3) that the structural equation easily may be expanded and made as complex as necessary for modeling longitudinal pin-point cover data, thus allowing the incorporation of the most important ecological processes in the state-space model without technical difficulties. Typically, there is considerable spatial variation in plant abundance, and this variation is modeled using the Pólya-Eggenberger distribution (a generalization of the beta-binomial distribution). To illustrate this method, longitudinal hierarchical pin-point data of Erica tetralix in wet Danish heathlands were analyzed, including and excluding autocorrelation and an environmental covariable in the state-space model. The pin-point plant cover data showed a significant decrease in the plant cover of E. tetralix in the period from 2004 to 2009, with an annual decrease of about $10 \%$ in the logit-transformed cover. The distribution of predicted plant cover at a given site the following year was calculated, including and excluding the information of an environmental covariable.
\end{abstract}

Key words: ecological monitoring; Erica tetralix; longitudinal pin-point cover data; state-space model; wet heathlands.

\section{INTRODUCTION}

The ability to perform robust trend analyses without losing information or statistical power is a fundamental requirement of ecological monitoring programs. Often, ecological monitoring data are sampled at significant costs, and it is important that as much relevant ecological information is retrieved from the data as possible. In the present study, I present a general method for analyzing trends in hierarchically sampled plant species abundance data using state-space models.

Plant abundance is often described by cover, i.e., the relative area covered by different plant species in a small plot. Plant cover is not biased by the size and distribution of individuals and is an important and often measured characteristic of the composition of plant communities (Kent and Coker 1992). The most common way to measure plant cover in herbaceous plant communities is to make a visual assessment of the relative area covered by the different species within a circle or a square (Kent and Coker 1992). However, an alternative more objective methodology, called the pinpoint method (or point-intercept method), has been widely employed (Levy and Madden 1933, Kent and Coker 1992). In a pin-point analysis, a frame with a fixed grid pattern is placed randomly above the

Manuscript received 17 August 2011; revised 17 January 2012; accepted 23 January 2012. Corresponding Editor: B. D. Inouye

${ }^{1}$ E-mail: cfd@dmu.dk vegetation, and a pin is inserted vertically through one of the grid points into the vegetation. The different species touched by the pin are recorded at each insertion, and the cover of the plant species is defined as the relative number of pin hits.

Plant cover data, e.g., when measured in biodiversity monitoring programs, are often sampled using a hierarchical sampling procedure, where several plots are sampled from a number of different sites. Furthermore, the abundance of different plant species generally displays aggregated spatial patterns within the site due to, e.g., the size of the plant, clonal growth, and limited seed dispersal. Plant cover data will typically be overdispersed relative to the binomial distribution (Pacala and Levin 1997, Herben et al. 2000, Stoll and Weiner 2000). Here, the spatial variation in plant abundance will be modeled using the Pólya-Eggenberger distribution, which allows for an augmented variance compared to a binomial distribution.

In the present study, I will advocate the use of statespace models as a versatile and robust method of analyzing longitudinal pin-point cover data (Clark and Bjørnstad 2004, Clark 2007, Damgaard et al. 2011, de Valpine 2011). The main advantages of using a statespace model are (1) that the observed variance is separated into sampling variance and the more interesting structural variance that are needed for quantifying prediction uncertainty, (2) that missing values or an unbalanced sampling design readily may be accounted for, and (3) that the structural equation easily may be 
expanded and made as complex as necessary for modeling longitudinal pin-point cover data, thus allowing the incorporation of the most important ecological processes in the state-space model without technical difficulties.

In order to demonstrate the method for analyzing trends in pin-point cover data, hierarchical longitudinal pin-point data of Erica tetralix in wet heathlands sampled in the Danish monitoring program NOVANA (Nielsen et al., in press) were chosen as a case study. In order to demonstrate how the state-space model may be easily extended and made more biological realistic I also investigated whether the trend in cover depends on the model-calculated atmospheric nitrogen deposition (Ellermann et al. 2007).

\section{Materials And Methods}

\section{Pin-point plant cover data}

Erica tetralix is a perennial subshrub, which is a characteristic plant species of humid, peaty or semipeaty heaths (Northern Atlantic wet heaths [EU habitat code 4010]; EU 2003). Twenty-three Danish sites with wet heathlands were monitored from 2004 to 2009. At each site, 20-60 plots were placed randomly, and in each plot the plant cover of higher plants was measured by the pin-point method in square frames of 16-grid points separated by $10 \mathrm{~cm}$ between each grid point (Nielsen et al., in press). The plots were revisited each year in the period with GPS accuracy $(<10 \mathrm{~m})$, although there were a number of missing values due to incomplete sampling over the years. The vegetation types of all sampled plots were classified according to the habitat classification system used for the European Habitat Directive (EU 2003) by the classification method outlined in Nygaard et al. (2009), and only the plots that were classified as wet heathland (EU habitat code 4010) in more than three years were used in the present analyses.

After this initial screening, only 15 sites had more than 20 records of the cover of E. tetralix, and these were selected for further analyses. At the 15 selected sites, there were a total of 1670 records. The number of selected plots varied both among sites and among years (the variation among years was due to missing values).

\section{The distribution of pin-point plant cover data}

Before discussing the trend analysis of pin-point data, it is necessary to consider the measurement of pin-point cover data, which is a binomial process where a single pin in the pin-point frame either hits or does not hit the plant species. The stochastic variable $y$ is defined as the number of pins out of $n$ pins in the frame that touch the plant species. Since the distribution of plant species typically is spatially aggregated, $y$ is assumed to be generated by a generalized binomial distribution (or Pólya-Eggenberger distribution) with probability parameter $q$ and intra-plot correlation parameter $\delta$ :

$$
\begin{aligned}
& f(y ; n, q, \delta) \\
& =\left(\begin{array}{l}
n \\
y
\end{array}\right) \frac{\phi\left(q\left(\frac{1}{\delta}-1\right), y\right) \phi\left(\frac{(1-q)(1-\delta)}{\delta}, n-y\right)}{\phi\left(\frac{1}{\delta}-1, n\right)}
\end{aligned}
$$

where the parameters are explained in Table 1 and $\varphi$ is the Pochhammer symbol, $\varphi(x, n)=\Gamma(x+n) / \Gamma(x)=(x+$ 1) $\ldots(x+n-1)$ (Damgaard 2008, 2009). The mean of the generalized binomial distribution is independent of $\delta ; E(y)=n q$, and $\operatorname{Var}(y)=n(1-q) q(1-\delta(1-n))$.

The probability density function of the Pólya-Eggenberger distribution (Eq. 1) is equal to the beta-binomial distribution $f(y ; n, v)=\operatorname{Binomial}(n, v)$, where

$$
v \sim \operatorname{Beta}\left(\frac{q}{\delta}-q, \frac{(1-q)(1-\delta)}{\delta}\right)
$$

but is somewhat more general in that negative intra-plot correlation is allowed (Qu et al. 1993).

\section{Trend analysis of hierarchical pin-point plant cover data}

It was decided to model the change in plant cover at the level of the site rather than at the level of the plots, since (1) the selected pin-point data are hierarchical with several plots within a site; (2) the different sites have separate histories and management practices, as well as environmental conditions (e.g., climate, base cation deposition, and nitrogen deposition) and are, consequently, not expected to have the same change in plant cover; (3) the data set is not complete and several plots were not sampled each year; (4) the position of the plots is only known with GPS certainty $(<10 \mathrm{~m})$. Furthermore, in order to separate the observed variance of the longitudinal pin-point plant cover data into sampling variance and the more interesting variance of the annual change in plant cover, the change in cover was analyzed using a state-space model, where the unknown mean cover at each site is modeled by a latent variable (Clark 2007, Bruus et al. 2010, Damgaard et al. 2011, de Valpine 2011). A state-space model consists of a structural equation, where the processes that control the change in the unknown mean cover over time are described, and a measurement equation, where the observations or measurements are coupled to the latent variables, i.e., the unknown mean cover at site $i$ at observation time $t$ (Fig. 1).

\section{Structural equation}

The structural equation consists of a deterministic part that describes the average change in cover and a stochastic part that describes the variation in the annual change. The unknown cover at site $i$ to the year $t$ is denoted $x_{i, t}$, and it is assumed that the average change in the logit-transformed cover $[\operatorname{logit}(x)=\log (x /[1-x])]$ is constant with independent and identical normally distributed variance $\sigma^{2}$ : 
TABLE 1. The parameters in the generalized binomial distribution (Eq. 1).

\begin{tabular}{cl}
\hline \hline Parameter & \multicolumn{1}{c}{ Description } \\
\hline$y$ & Number of times the plant species is hit in a pin point frame. \\
$n$ & Number of grid points in the grid point frame $(n=16)$. \\
$q$ & Expected plant cover. \\
& The intra-plot correlation parameter, which is bounded between $-\min [q /(n-1-q),(1-q) /(n-1)]$ and 1, \\
& measures the spatial aggregation of the plant species as the correlation between the outcomes of successive \\
& Bernoulli trials (Qu et al. 1993). For plant species that tend to be spatially aggregated, the number of hits \\
& within a pin-point frame is positively correlated. In this case $(\delta>0)$, the variance of the number of hits will \\
& be augmented relative to the binomial distribution. The hypothesis of no correlation (binomially distributed \\
& hits) may be tested in a likelihood ratio test.
\end{tabular}

$$
\operatorname{logit}\left(x_{i, t}\right)=\alpha+\operatorname{logit}\left(x_{i, t-1}\right)+\varepsilon_{i, t}
$$

where $\alpha$ is the average yearly change in plant cover and $\varepsilon_{i, t} \sim \mathcal{N}\left(0, \sigma^{2}\right)$.

The above structural model (Eq. 2) may be extended in various ways, e.g., by assuming that the yearly annual change in plant cover depends on the level of an environmental variable $(z)$ :

$$
\operatorname{logit}\left(x_{i, t}\right)=\alpha+\beta\left(z_{i}-\bar{z}\right)+\operatorname{logit}\left(x_{i, t-1}\right)+\varepsilon_{i, t} .
$$

In the present case study, the environmental variable $(z)$ is the model-calculated atmospheric nitrogen deposition (Ellermann et al. 2007), and $\beta$ is a regression coefficient.

\section{Measurement equation}

Since the latent variables were modeled independently for each site, it is assumed that the number of pin-point hits in a given year at a site was independent and identically distributed from a generalized binomial distribution (Eq. 1):

$$
f_{y}\left(y_{i, p, t} ; n, 0, x_{i, t}, \delta\right)
$$

where $y_{i, p, t}$ is the observed number of hits at site $i$ in plot $p$ in year $t, n$ is the number of grid points in the pin-point frame, and $\delta$ is the intra-plot correlation parameters that measure the degree of spatial aggregation, which is assumed to be a characteristic feature of a plant species and not to vary among sites (Table 1).

Alternatively, in order to account for possible autocorrelation among the pin-point observations from the same plot among years, the probability of observing $y$ hits may also be modeled by

$$
f_{y}\left(y_{i, p, t} ; n, 0, b\left(x_{i, t}+\rho\left(y_{i, t-1} \times n^{-1}-x_{i, t-1}\right)\right), \delta\right)
$$

where $y_{i, p, t}$ is an autocorrelation parameter and $b(\cdot)$ is an identity function that bound $q$ in the interval $[1 / 4 n, 1-$ $(1 / 4 n)]$. In this study of E. tetralix, where $n=16$, the interval is $[1 / 64,63 / 64]$. The bounding function is needed because $q$ has to take values in the open interval $(0,1)$, and $1 / 4 n$ was chosen as a reasonable margin away from 0 or 1 .

\section{Likelihood function}

The integrated likelihood function of the full statespace model may be specified by multiplying a number of relatively simple conditional likelihood expressions
(Clark 2007), i.e., using a first-order Markov assumption. The likelihood function

$$
\prod_{i} p\left(x_{i, 1}, x_{i, 2} \ldots x_{i, n}\right)
$$

may be described by

$$
\prod_{i} p\left(x_{i, n} \mid x_{i, n-1}\right) \ldots p\left(x_{i, 2} \mid x_{i, 1}\right) p\left(x_{i, 1}\right)
$$

where $p\left(x_{i, 1}\right)$ is a prior distribution of the latent variable. The likelihood function of the measurement equations may be expressed by

$$
\prod_{i, p, t} p\left(y_{i, p, t} \mid x_{i, t}\right)
$$

and the likelihood function of the parameters by $p(\theta \mid \operatorname{prior}[\theta])$.

\section{Estimation, statistical inference, and model comparison}

The model was parameterized using numerical Bayesian methods, where the joint posterior distribution of the parameters and the latent variables were calculated using MCMC (Markhov chain Monte Carlo) Metropolis-Hastings simulations (Carlin and Louis 1996) with a multivariate normal candidate distribution, and using a MCMC run of 100000 iterations with a burn-in period of 1000 iterations. The prior distributions of all parameters were assumed to be uniformly distributed as improper priors, except the following parameters that were assumed to be uniformly distributed in a specified interval: $-2<\alpha<2,-2<\beta<2,0.01<\delta<0.99,-1<$

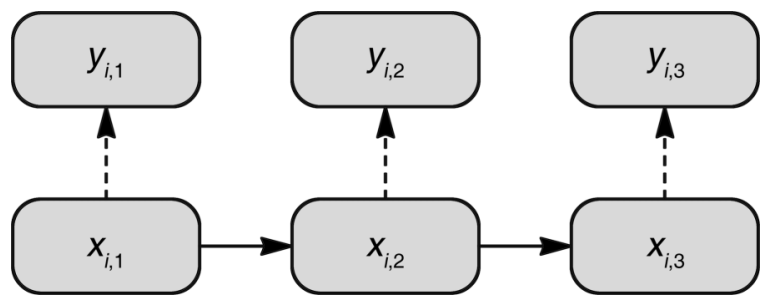

FIG. 1. Conceptual graph of the state-space model for site $i$ in three years, where the latent variable for the unknown plant cover at site $i$ at the year $t$ is denoted $x_{i, t}$ and $y_{i, t}$ is the observed number of hits at site $i$ in year $t$. The solid arrows indicate the structural equations, and the dashed arrows indicate the measurement equations. 


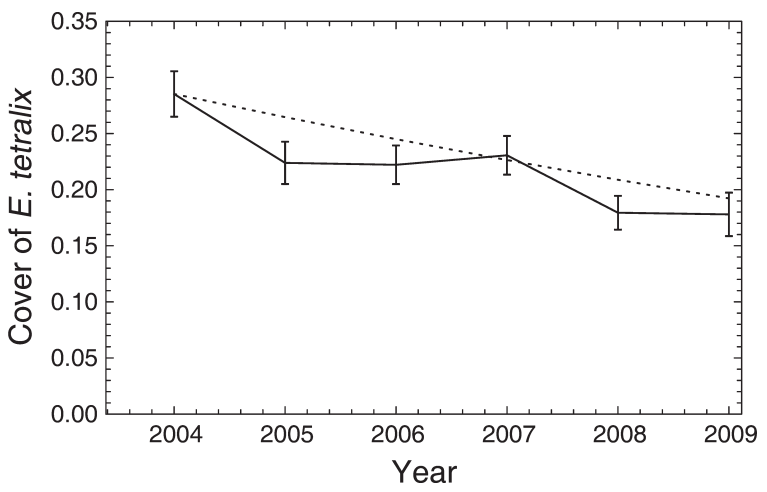

FIG. 2. The observed changes in the mean plant cover of Erica tetralix for all measured plots during the period. The bars denote the standard error of the mean for all plots. The dotted line is the estimated change in cover using the median of the marginal distribution of $\alpha$ when fitted with model M2 at a hypothetical site with a mean cover in 2004 that is equal to the mean of all the plots (see Table 2 for models).

$\rho<1$, and $\sigma$ that was assumed to uniformly distributed and larger than 0.01 . The latent variables were assumed to be uniformly distributed within $[1 / 4 n, 1-(1 / 4 n)]$. Plots of the sampling chains of all parameters and latent variables were inspected in order to check the mixing properties of the used sampling procedure. Additionally, the overall fitting properties of the model were checked by inspecting the covariance matrix of all parameters and the regularity and shape of the marginal distribution of all parameters, as well as the distribution of the deviance. The efficiency of the MCMC procedure was assessed by inspecting the evolution in the deviance as well as calculating autocorrelation and acceptance rates of the MCMC chain.

Statistical inferences on the parameters may be based on the marginal posterior distribution of the parameters by the $95 \%$ credibility intervals of the parameters. Different models may be compared using the deviance information criterion (DIC), which is the mean deviance
$[-2 \log \mathcal{L}(y \mid \theta)]$ of the MCMC run plus the "effective" number of free parameters that are also calculated from the MCMC run (Spiegelhalter et al. 2002). Similarly, as for Akaike information criterion (AIC), the preferred model is the one with the minimum DIC value.

All calculations were done with Mathematica (version 7.0; Wolfram Research, Champaign, Illinois, USA) see Appendix A for the notebook file, data, results, diagnostic graphs, and more information.

\section{Results AND Discussion}

The plant cover of E. tetralix in wet heathlands decreased $36 \%$ from a mean cover of $28 \%$ in 2004 to a mean cover of $18 \%$ in 2009 (Fig. 2). The hierarchical pint-point cover data was fitted to the four combinations of the structural and measurement equations (Eqs. 2-5), where the environmental variable $(z)$ was the modelcalculated atmospheric nitrogen deposition (Ellermann et al. 2007). The MCMC sampling chains of all four combinations of the structural and measurement equations (Eqs. 2-5) exhibited satisfactory properties (see Mathematica notebook in the Supplement).

The joint posterior distributions of the parameters of the four fitted models are shown in Table 2. The average yearly change as measured by $\alpha$ was significantly negative for all four models (Table 2, $P<0.003$ ), with an annual decrease in the logit-transformed plant cover of about $10 \%$, thus corroborating the more intuitive result of the decreasing mean plant cover of E. tetralix in Fig. 2.

In the present case, the positions of plots for the repeated pin-point measurements were only known with GPS certainty, thus it was decided to model the effect of autocorrelation. As expected, the deviation information criterion (DIC) of the four models (Table 2) suggested that the fit of the state-space model was superior when possible autocorrelation among the pin-point observations from the same plot among years was included in the model (measurement Eq. 5), and the estimated median values of $\rho$ were 0.47 for both models where autocorrelation was included (M2 and M4 in Table 2).

TABLE 2. The deviance information criterion (DIC) and the marginal posterior distribution of the parameter of interests $\{2.5 \%$ percentile, $50 \%$ percentile, $97.5 \%$ percentile\} calculated for the different state-space models (Eqs. 2-5).

\begin{tabular}{|c|c|c|c|c|c|c|}
\hline State-space model & DIC & $\alpha$ & $\beta$ & $\sigma$ & $\rho$ & $\delta$ \\
\hline $\begin{aligned} \text { M1: } & \Delta \operatorname{logit}\left(x_{i, t}\right) \\
= & \alpha+\varepsilon_{i, t} \dagger\end{aligned}$ & 6289.78 & $\begin{array}{c}\{-0.162,-0.102 \\
-0.045\}\end{array}$ & - & $\begin{array}{l}\{0.055,0.113, \\
0.225\}\end{array}$ & - & $\begin{array}{c}\{0.430,0.454, \\
0.478\}\end{array}$ \\
\hline $\begin{aligned} \text { M2: } & \Delta \operatorname{logit}\left(x_{i, t}\right) \\
& =\alpha+\varepsilon_{i, t}\end{aligned}$ & 6105.03 & $\begin{array}{l}\{-0.167,-0.103 \\
\quad-0.038\}\end{array}$ & - & $\begin{array}{l}\{0.054,0.142 \\
0.275\}\end{array}$ & $\begin{array}{l}\{0.407,0.466, \\
0.526\}\end{array}$ & $\begin{array}{c}\{0.393,0.417, \\
0.442\}\end{array}$ \\
\hline M3: $\begin{aligned} & \Delta \operatorname{logit}\left(x_{i, t}\right) \\
= & \alpha+\beta\left(z_{i}-\bar{z}\right)+\varepsilon_{i, t}^{\dagger}\end{aligned}$ & 6245.77 & $\begin{array}{l}\{-0.159,-0.109 \\
\quad-0.045\}\end{array}$ & $\begin{array}{l}\{-0.012,0.010 \\
0.034\}\end{array}$ & $\begin{array}{l}\{0.031,0.095 \\
\quad 0.215\}\end{array}$ & - & $\begin{array}{l}\{0.430,0.455 \\
0.479\}\end{array}$ \\
\hline M4: $\begin{aligned} \Delta \operatorname{logit}\left(x_{i, t}\right) \\
\quad=\alpha+\beta\left(z_{i}-\bar{z}\right)+\varepsilon_{i, t}\end{aligned}$ & 6098.17 & $\begin{array}{l}\{-0.155,-0.094, \\
\quad-0.030\}\end{array}$ & $\begin{array}{l}\{-0.007,0.016 \\
\quad 0.040\}\end{array}$ & $\begin{array}{l}\{0.053,0.134 \\
0.251\}\end{array}$ & $\begin{array}{l}\{0.411,0.469 \\
0.528\}\end{array}$ & $\begin{array}{l}\{0.393,0.416 \\
0.441\}\end{array}$ \\
\hline
\end{tabular}

Notes: The latent variable for the unknown plant cover at site $i$ at the year $t$ is denoted $x_{i, t}$. The environmental variable $(z)$ was the model-calculated atmospheric nitrogen deposition (Ellermann et al. 2007), $\alpha$ is the average yearly change in plant cover, $\beta$ is a regression coefficient that measure the effect of nitrogen deposition, $\sigma$ is the estimates process error, $\rho$ is an auto-correlation coefficient, and $\delta$ is the intra-plot correlation parameter.

$\dagger$ Without autocorrelation, $\rho=0$, measurement Eq. 4.

$\$$ With autocorrelation, measurement Eq. 5. 
Including autocorrelation in the model had a slight effect on the estimated intra-plot correlation of the pinpoint hits; the median values of $\delta$ decreased from 0.45 in the two state-space models without autocorrelation (M1 and M3 in Table 2) to 0.42 in the two state-space models with autocorrelation (M2 and M4 in Table 2). If the position of the plot had been permanently marked, then it probably would have been more efficient to model the change in cover at each plot.

I used a bounded identity autocorrelation function, however, other autocorrelation functions may be relevant, e.g., a bounded sigmoid function of cover. Qualitatively similar results were obtained with a sigmoid autocorrelation function, but with a worse model fit, i.e., considerably higher DIC values (results not shown). Due to the relatively worse fit of the sigmoid autocorrelation function and in order to avoid unnecessary complexity, I suggest using a bounded identity autocorrelation function.

The fit of the state-space model was improved when the model-calculated atmospheric nitrogen deposition (Ellermann et al. 2007) was included as an explanatory variable (M3 and M4 in Table 2), although the effect of nitrogen deposition on the yearly change in plant cover was nonsignificant (the $95 \%$ credibility interval of $\beta$ included zero; Table 2).

The use of DIC for comparison of models that are fitted using MCMC approaches is still a relatively new method, and there is still discussion on the use of the DIC, especially in the comparison of mixed models. However, there seems to be consensus that DIC is a useful model selection criterion when there is a clear focus on the parameter of interest, i.e., the parameters that are reported in Table 2 (Spiegelhalter et al. 2002, Celeux et al. 2006).

Generally, state-space models are suitable for dealing with unbalanced hierarchical data, as in this methodological study, since the estimated parameters of interest are not influenced by irregular data sampling (Clark and Bjørnstad 2004). Furthermore, the method allows separation of process and sampling variance, which enables ecological predictions with a known degree of uncertainty (Clark 2007). Thus, it is possible to predict the expected distribution of the plant cover of E. tetralix in year $t$ at a specific site from a known plant cover in year $t-1$ by randomly drawing from the joint distribution of the parameters:

$$
\operatorname{Normal}\left(\left[\operatorname{logit}\left(x_{t}\right)\right]+\Delta, \sigma^{2}\right)
$$

where $\Delta$ is drawn from the expected annual change of the logit-transformed cover in the structural equations (Eqs. 2 or 3). For example, using the results of statespace model 2 (Table 2), if the plant cover of E. tetralix at a site is $20 \%$ in 2009 , then the median predicted plant cover at the site in 2010 is 0.18 , with a $95 \%$ credibility interval of $0.14-0.24$. Furthermore, if we know that the amount of nitrogen deposition at the site is 10 $\mathrm{kg} \cdot \mathrm{ha}^{-1} \cdot \mathrm{yr}^{-1}$ and use the results of state-space model 4 (Table 2), then the median predicted plant cover at the site in 2010 is 0.17 , with a $95 \%$ credibility interval of $0.13-0.22$.

Bayesian methods have been criticized for relying on subjectively determined prior distributions of the parameters; data cloning (Lele et al. 2007, Ponciano et al. 2009) has been proposed to reduce the importance of the prior distribution. For the present analysis, normally distributed uninformative prior distributions were tried with no apparent effect on the results. That the present results are insensitive to various uninformative prior distributions is expected, since the model is fitted to many data (1670 records), and for the same reason data cloning techniques are not expected to change the reported conclusion in the present analysis. However, if fewer data are available, data cloning may be an attractive alternative to the fitting method presented here.

Erica tetralix is a characteristic plant species of wet heathlands, and the observed significant decrease in plant cover of E. tetralix is of considerable concern with respect to the requirements of the European Union habitat directive (EU 1992). It is outside the scope of this study to explore the reasons for the decrease in plant cover, but the possible effects of acidification, nitrogen deposition, as well as changes in hydrology and management practice on the cover of E. tetralix are investigated more fully elsewhere (Strandberg et al. 2011, 2012).

\section{Literature Cited}

Bruus, M., K. E. Nielsen, C. Damgaard, B. Nygaard, J. Fredshavn, and R. Ejrnæs. 2010. Terrestriske naturtyper 2008. DMU, Aarhus Universitet, Silkeborg, Denmark.

Carlin, B. P., and T. A. Louis. 1996. Bayes and empirical Bayes methods for data analysis. Chapman and Hall, London, UK.

Celeux, G., F. Forbes, C. P. Robert, and D. M. Titterington. 2006. Deviance information criteria for missing data models. Bayesian Analysis 1:651-674.

Clark, J. S. 2007. Models for ecological data. Princeton University Press, Princeton, New Jersey, USA.

Clark, J. S., and O. N. Bjørnstad. 2004. Population time series: process variability, observation errors, missing values, lags, and hidden states. Ecology 85:3140-3150.

Damgaard, C. 2008. Modelling pin-point plant cover data along an environmental gradient. Ecological Modelling 214:404-410.

Damgaard, C. 2009. On the distribution of plant abundance data. Ecological Informatics 4:76-82.

Damgaard, C., B. Nygaard, R. Ejrnæs, and J. Kollmann. 2011. State-space modeling indicates rapid invasion of an alien shrub in coastal dunes. Journal of Coastal Research 27:595599.

de Valpine, P. 2011. Frequentist analysis of hierarchical models for population dynamics and demographic data. Journal of Ornithology. http://dx.doi.org/10.1007/s10336-010-0642-5

Ellermann, T., H. V. Andersen, R. Bossi, J. Christensen, L. M. Frohn, C. Geels, K. Kemp, P. Løfstrøm, B. B. Mogensen, and C. Monies. 2007. Atmospheric deposition 2006. NOVANA. National Environmental Research Institute, Aarhus University, Silkeborg, Denmark. [In Danish.]

EU. 1992. Council Directive 92/43/EEC of 21 May 1992 on the conservation of natural habitats and of wild fauna and flora.

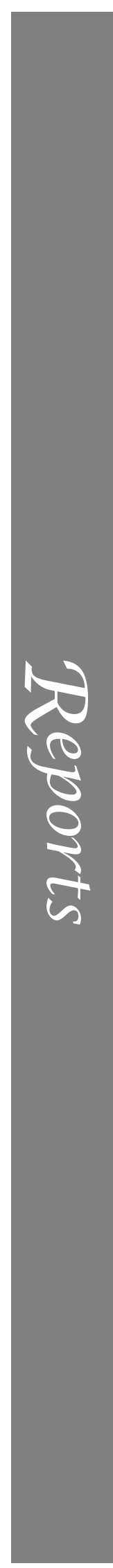


Official Journal of the European Communities. http://eur-lex. europa.eu/LexUriServ/LexUriServ.do?uri=OJ:L:1992:206: 0007:0050:EN:PDF

EU. 2003. Interpretation manual of European Union habitats. Natura 2000. European Commission. European Commission, DG Environment, Nature and Biodiversity, Brussels, Belgium.

Herben, T., H. J. During, and R. Law. 2000. Spatio-temporal patterns in grassland communities. Pages 48-64 in $\mathrm{U}$. Dieckmann, R. Law, and J. A. J. Metz, editors. The geometry of ecological interactions: simplifying spatial complexity. Cambridge University Press, Cambridge, UK.

Kent, M., and P. Coker. 1992. Vegetation description and analysis. Belhaven Press, London, UK.

Lele, S. R., B. Dennis, and F. Lutscher. 2007. Data cloning: easy maximum likelihood estimation for complex ecological models using Bayesian Markov Monte Carlo methods. Ecology Letters 10:551-563.

Levy, E. B., and E. A. Madden. 1933. The point method of pasture analyses. New Zealand Journal of Agriculture 46:267-279.

Nielsen, K. E., J. L. Bak, M. Bruus Pedersen, C. Damgaard, R. Ejrnæs, J. R. Fredshavn, B. Nygaard, F. Skov, B. Strandberg, and M. Strandberg. In press. Danish monitoring program of vegetation and chemical plant and soil data from non-forested terrestrial habitat types. Biodiversity and Ecology.

Nygaard, B., R. Ejrnæs, A. Baattrup-Pedersen, and J. Fredshavn. 2009. Danske plantesamfund i moser og enge-vegetation, $\emptyset$ kologi, sårbarhed og beskyttelse. Faglig rapport fra DMU nr. 728. DMU, Aarhus Universitet, Silkeborg, Denmark.
Pacala, S., and S. A. Levin. 1997. Biological generated spatial pattern and the coexistence of competing species. Pages 204232 in D. Tilman and P. Kareiva, editors. Spatial ecology. The role of space in population dynamics and interspecific interactions. Princeton University Press, Princeton, New Jersey, USA.

Ponciano, J. M., M. L. Taper, B. Dennis, and S. R. Lele. 2009. Hierarchical models in ecology: confidence intervals, hypothesis testing, and model selection using data cloning. Ecology 90:356-362.

Qu, Y., T. Greene, and M. R. Piedmonte. 1993. Symmetric Bernoulli distributions and generalised binomial distributions. Biometrical Journal 35:523-533.

Spiegelhalter, D. J., N. G. Best, B. P. Carlin, and A. van der Linde. 2002. Bayesian measures of model complexity and fit. Journal of the Royal Statistical Society B 64:583-639.

Stoll, P., and J. Weiner. 2000. A neighborhood view of interactions among individual plants. Pages 11-27 in U. Dieckmann, R. Law, and J. A. J. Metz, editors. The geometry of ecological interactions. Cambridge University Press, Cambridge, UK.

Strandberg, M., C. Damgaard, H. J. Degn, J. L. Bak, and K. E. Nielsen. 2012. Evidence for acidification-driven ecosystem collapse of Danish Erica tetralix wet heathland. Ambio. http://dx.doi.org/ 10.1007/s13280-012-0251-z

Strandberg, M., K. E. Nielsen, C. Damgaard, and H. J. Degn. 2011. Status og plejemuligheder for klokkelyngdomineret våd hede på Borris Hede. Aarhus Universitet, Silkeborg, Denmark.

\section{Supplemental Material}

\section{Supplement}

A Mathematica notebook file with data, results, diagnostic graphs, and more information (Ecological Archives E093-110-S1). 